\title{
Pharmacopuncture for Cancer Care: A Systematic Review
}

\author{
Soyeon Cheon, ${ }^{1,2}$ Xiuyu Zhang, ${ }^{1,2}$ In-Seon Lee, ${ }^{1,2}$ Seung-Hun Cho, ${ }^{3}$ \\ Younbyoung Chae, ${ }^{1,2}$ and Hyangsook Lee ${ }^{2}$ \\ ${ }^{1}$ Department of Korean Medical Science, Graduate School, Kyung Hee University, Kyung Hee Dae-ro 26, Dongdaemun-gu, \\ Seoul 130-701, Republic of Korea \\ ${ }^{2}$ Acupuncture and Meridian Science Research Center, College of Korean Medicine, Kyung Hee University, Kyung Hee Dae-ro 26, \\ Dongdaemun-gu, Seoul 130-701, Republic of Korea \\ ${ }^{3}$ Hospital of Korean Medicine, Kyung Hee University Medical Center, Kyung Hee Dae-ro 23, Dongdaemun-gu, \\ Seoul 130-701, Republic of Korea
}

Correspondence should be addressed to Hyangsook Lee; erc633@khu.ac.kr

Received 25 February 2014; Accepted 28 March 2014; Published 12 May 2014

Academic Editor: Yoshiharu Motoo

Copyright (C) 2014 Soyeon Cheon et al. This is an open access article distributed under the Creative Commons Attribution License, which permits unrestricted use, distribution, and reproduction in any medium, provided the original work is properly cited.

\begin{abstract}
Background. Pharmacopuncture, injection to acupoints with pharmacological medication or herbal medicine, is a new acupuncture therapy widely available in Korea and China for cancer-related symptoms. However, the evidence is yet to be clear. Objective. To determine pharmacopuncture's effectiveness on cancer-related symptoms. Methods. Eleven databases were searched for randomized controlled trials of pharmacopuncture in cancer patients. The Cochrane risk of bias (ROB) assessment tool was used for quality assessment. Results. Twenty-two studies involving 2,459 patients were included. Five trials of chemotherapy-induced nausea and vomiting (CINV) underwent meta-analysis. Pharmacopuncture significantly relieved severity of CINV compared with control group ( 3 trials, risk ratio $(\mathrm{RR}) 1.28,95 \%$ confidence interval $(\mathrm{CI})=1.14-1.44)$. The frequency of CINV was also significantly reduced with pharmacopuncture ( 2 trials, RR $2.47,95 \% \mathrm{CI}=2.12-2.89$ ). Seventeen trials studied various symptoms, and in most studies, pharmacopuncture significantly relieved pain, ileus, hiccup, fever, and gastrointestinal symptoms and improved quality of life in various cancer patients. ROB was generally high. Conclusion. It may be suggested with caution that pharmacopuncture may help various symptom relief in cancer patients, but it is hard to draw a firm conclusion due to clinical heterogeneity and high ROB of the included studies, hence warranting further investigation.
\end{abstract}

\section{Introduction}

Cancer is a well-known health problem worldwide. In 2008, GLOBOCAN [1] estimated about 12.7 million cancer cases worldwide, and a recent study made estimates of more than 1.6 million new cases in USA alone for 2013 [2]. More recent report from World Health Organization (WHO) is estimating that global incidence of cancer will rise up to 22 million per year within the next two decades [3]. Although there have been significant advances in the field of cancer treatments in the past decades $[4,5]$, effective management of cancer and its related symptoms still leaves much to be desired, and moreover, adverse events (AEs) that come along in the course of cancer treatment are another problem.
The frequently experienced AEs associated with cancer treatment, such as pain, nausea and vomiting, fatigue, or constipation, lead patients and researchers to seek new approaches, and among various options available, there is complementary and alternative medicine (CAM) as one approach $[6,7]$. Among various CAM therapies, pharmacopuncture, also known as acupoint injection, herbal acupuncture, aqua acupuncture, or aquapuncture, that is, pharmacological medication or purified herbal medicine injected to acupoints, is a new acupuncture therapy that combines acupuncture therapy and medication. It is now widely used in China and Korea for a range of symptoms including cancerrelated symptoms [8], and an Australian study reported that it induces higher de-qi sensation compared to traditional 
acupuncture, which may be an indication that pharmacopuncture could provide stronger clinical response than traditional acupuncture [9]. Also, some studies reported that pharmacopuncture in addition to manual acupuncture produced better clinical outcomes, such as significant improvement in pain and function for patients with herniated intervertebral disc $[10,11]$, or significantly less pain and shorter duration of pain for patients with postauricular pain from peripheral facial paralysis [12], compared with manual acupuncture alone. Although evidence for its efficacy is piling up [13-15], no systematic reviews for assessing pharmacopuncture for cancer care have been published yet.

Therefore, the aim of this review was to critically summarize and assess the current evidence from randomized controlled trials (RCTs) that investigated pharmacopuncture's effectiveness on cancer-related symptoms and to help clinicians and patients make informed decision making.

\section{Methods}

2.1. Data Sources and Searches. We searched for RCTs and systematic reviews of pharmacopuncture in cancer care. Electronic searches were conducted in PubMed, Embase, the Cochrane Central Register of Controlled Trials (CENTRAL), Cumulative Index to Nursing and Allied Health Literature (CINAHL), Chinese National Knowledge Infrastructure (CNKI), and related Korean databases including KoreaMed, KMbase, Riss4U, KISS, OASIS, and DBPIA from inception through March 2013. Also, trial registries (http://www .controlled-trials.com/, http://www.clinicaltrials.gov/) were searched to find any relevant ongoing or unpublished trials. No language restriction was imposed. Search strategy used for PubMed was as follows: ((pharmacopuncture*[All Fields] OR "herbal acupuncture" [All Fields] OR "aqua acupuncture" [All Fields] OR aquapuncture* [All Fields] OR "acupoint injection" [All Fields]) AND (Neoplasms [MeSH] OR Neoplasms* [TI] OR Cancer*[TI] OR Tumor*[TI] OR Tumour* ${ }^{*}$ TI] OR Carcinoma [MeSH] OR Carcinoma* [TI] OR Adenocarcinoma [MeSH] OR Adenocarcinoma*[TI] OR adenomatous [TI] OR Lymphoma [MeSH] OR lymphom* [TI] OR lymphedema* [TI] OR Sarcoma [MeSH] OR Sarcoma*[TI] OR “Antineoplastic agents" [MeSH] OR antineoplas $^{*}$ [TI] OR ((adenom* ${ }^{*}$ TI] OR adenopath* [TI]) AND malignant*[TI]))) AND (("Meta-Analysis as Topic" [Mesh] OR "Meta-Analysis" [PT] OR (meta [TIAB] AND analys ${ }^{*}[\mathrm{TIAB}]$ ) OR metaanalys ${ }^{*}$ [TIAB] OR (systematic [TIAB] AND (review* ${ }^{*}$ [TIAB] OR overview* ${ }^{*}$ [TIAB])) OR "Review Literature as Topic" [Mesh] OR Cochrane [TIAB] OR embase [TIAB] OR psychlit [TIAB] OR psyclit [TIAB] OR psychinfo [TIAB] OR psycinfo [TIAB] OR cinahl [TIAB] OR cinhal [TIAB] OR "Science citation index" [TIAB] OR bias [TIAB] cancerlit [TIAB] (reference [TIAB] AND list*[TIAB]) OR bibliograph*[TIAB] OR (hand [TIAB] AND search*[TIAB]) OR "relevant journals" [TIAB] OR (manual [TIAB] AND search*[TIAB]) OR (("selection criteria” [TIAB] OR “data extraction" [TIAB]) AND review [PT]) NOT (Comment [PT] OR Letter [PT] OR Editorial [PT] OR (Animals [MeSH] NOT (Animals [MeSH] AND
Human $[\mathrm{MeSH}]))$ )) OR ("randomized controlled trial" [PT] OR "controlled clinical trial" [PT] OR random* [TIAB] OR placebo [TIAB] OR "drug therapy" [Subheading] OR trial [TIAB] OR groups [TIAB] NOT (Animals [MeSH] NOT Humans $[\mathrm{MeSH}]))$ ). For individual search of each database, slight modifications were applied to the above strategy.

2.2. Study Selection. Search results were screened by one author (SC) based on the title and the abstract first and then were selected for the final analysis by two authors (SC and $\mathrm{XZ}$ ). To be included in the systematic review, studies should involve and randomly allocate cancer patients to a pharmacopuncture group or a control group. If there are other cointerventions such as chemotherapy, radiotherapy, and/or other palliative or supportive care, they should be given identically to both groups. Studies were included if they reported clinical symptom improvement, that is, studies reporting laboratory findings only were excluded.

2.3. Data Extraction and Assessment of Risk of Bias (ROB). The following information was subject to collecting data: first author's name, country, year of publication, number of participants, types of cancer, details of interventions for experimental and control groups, cointerventions, outcome measures, results for the outcome measures, and reported AEs associated with pharmacopuncture.

$\mathrm{ROB}$ was assessed independently by two authors (SC and I-SL) using the modified ROB assessment tool from the Cochrane Handbook [39]. The criteria consist of 6 items that might be related to selection bias (random sequence generation and allocation concealment), performance bias (blinding of participants and personnel), detection bias (blinding of outcome assessment), attrition bias (incomplete outcome data), and reporting bias (selective outcome reporting). Any discrepancies between the two reviewers were resolved by a thorough discussion with a corresponding author (HL) until consensus was reached.

2.4. Data Analysis. Studies were combined in the analysis according to the outcome measure, intervention type, and/or control type. When there was more than one control groups within a study, we chose the control group that administered the identical drug that was used for the intervention group over the other control group that used different type of drug, because that would make the comparison between the two groups limited to the efficacy of injection type only, for example, pharmacopuncture or intravenous (IV) injection. Data were pooled using a random effects model. The impact of pharmacopuncture on dichotomous outcomes was expressed with risk ratio (RR) with $95 \%$ confidence intervals (CI) and the effect of pharmacopuncture on continuous data was expressed with mean difference (MD) with 95\% CI. To generate forest plots of pooled estimates with 95\% CI, Review Manager (Version 5.2 Copenhagen: The Nordic Cochrane Centre, The Cochrane Collaboration, 2012) was used. Visual inspection of forest plots and a chi-squared test with a $P$ value less than 0.1 were used to assess heterogeneity among studies. The $I^{2}$ test was also used to quantify inconsistencies 


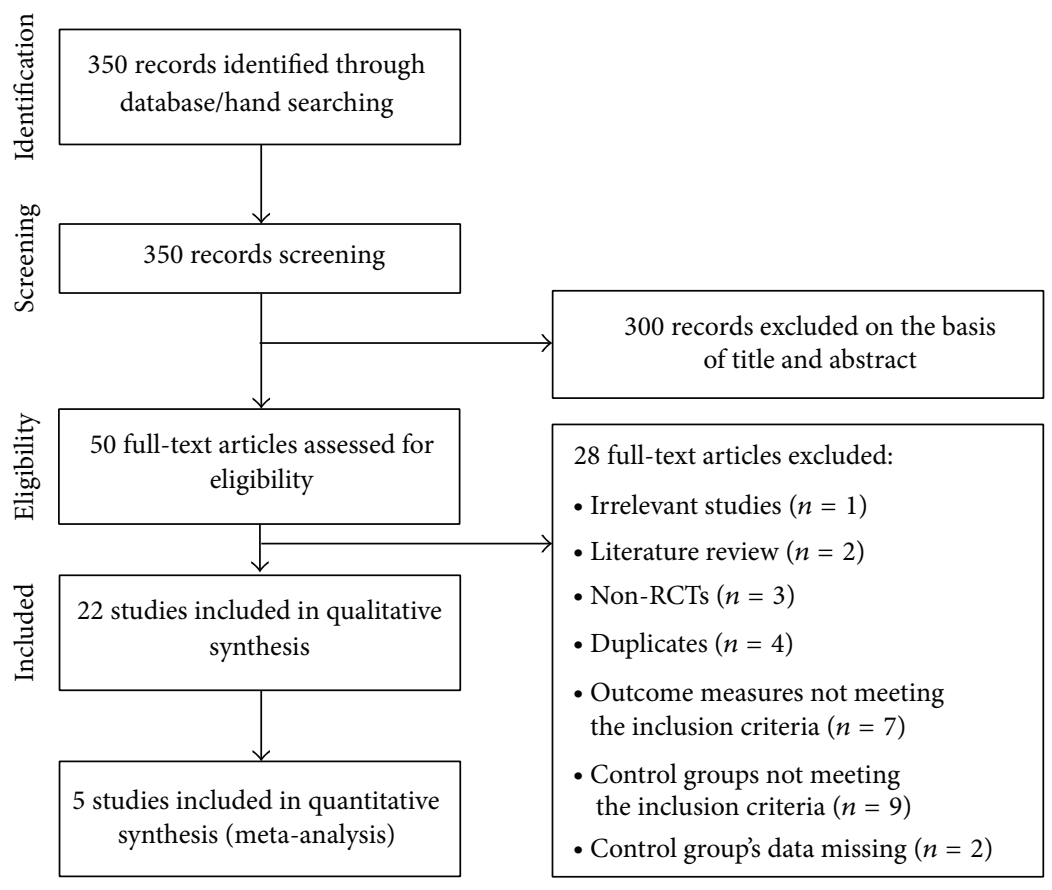

FIGURE 1: Flow diagram for selection of studies. RCTs: randomized controlled trials.

among the pooled studies and if important heterogeneity existed, we explored the reasons for heterogeneity. The $I^{2}$ statistic indicates the proportion of variability among studies that is not explained by chance alone and we considered that an $I^{2}$ value of $50 \%$ or more indicated a substantial level of heterogeneity [40, 41]. When data pooling was deemed misleading due to clinical heterogeneity and a small number of studies, a qualitative synthesis was undertaken.

\section{Results}

3.1. Study Selection and Description. A total of 350 articles were initially identified using our search strategy. Threehundred articles were excluded based on the title and abstract, leaving 50 articles to be screened with full text. Of these, 22 studies involving 2,459 participants met all the inclusion criteria and were included in our review (Figure 1). All 22 studies originated from China, and among them, one study [26] was published in English, and the others were all in Chinese. One article [18] was a doctoral dissertation and 21 were published in peer-reviewed journals. Seven studies recruited participants of certain cancer types such as late gastric cancer, ovarian cancer, hematologic cancer, rectal cancer, and upper gastrointestinal (GI) cancer [19, $24,26,27,31,32,36]$. The other 15 studies involved participants with various types of cancer [16-18, 20-23, 25, $28,29,33-35,37,38]$. We could categorize the topics of the included studies under 5 individual symptoms, that is, pain (8 studies [16-23]), chemotherapy-induced nausea and vomiting (CINV, 6 studies [24-29]), ileus (two studies $[31,32]$ ), hiccup (two studies [33, 34]), and fever (two studies $[35,36])$. In addition, there were two other categories which were quality of life (QOL) [37] and GI symptoms in general [38]. For intervention, 4 studies [18, 23, 28, 37] used Chinese herbal medicine injection at the acupoints, and 18 studies used pharmacological medication injection. All studies compared pharmacopuncture with conventional medication and among them, 4 studies $[19,26,27,29]$ had two control groups. All 4 of them compared pharmacopuncture with non-pharmacopuncture of an identical drug that was used in the intervention group for one control; for another control group, two studies $[19,26]$ used manual acupuncture, and two others $[27,29]$ used injection of different pharmacological medication. Within the other 18 studies that had only one control group, while all of them used pharmacological medication, 7 studies used identical drug that was used in the intervention group and 11 studies used different kind of drugs.

Characteristics of 8 studies of pain are summarized in Table 1. Five studies [19-23] were of sample sizes over 100 and three studies [16-18] had a smaller size between 40 and 52. Six studies were on CINV and their characteristics are summarized in Table 2. Sample sizes ranged from 51 to 480 [24-29]. Various control groups were used: intramuscular (IM) injection or manual acupuncture (one study), IV injection (4 studies), and oral medication (one study). Characteristics of the other included studies for symptoms of ileus, hiccup, fever, QOL, and GI symptoms are tabulated in Table 3. Three studies for ileus and QOL had a sample size between 108 and 160 [31, 32, 37], while the other 5 studies' sample size varied from 38 to 58 [33-36, 38]. Study designs were pharmacopuncture versus IM injection (5 studies), pharmacopuncture versus IM injection or oral intake (one study), pharmacopuncture combined with routine postoperative therapy versus routine postoperative therapy alone (one 


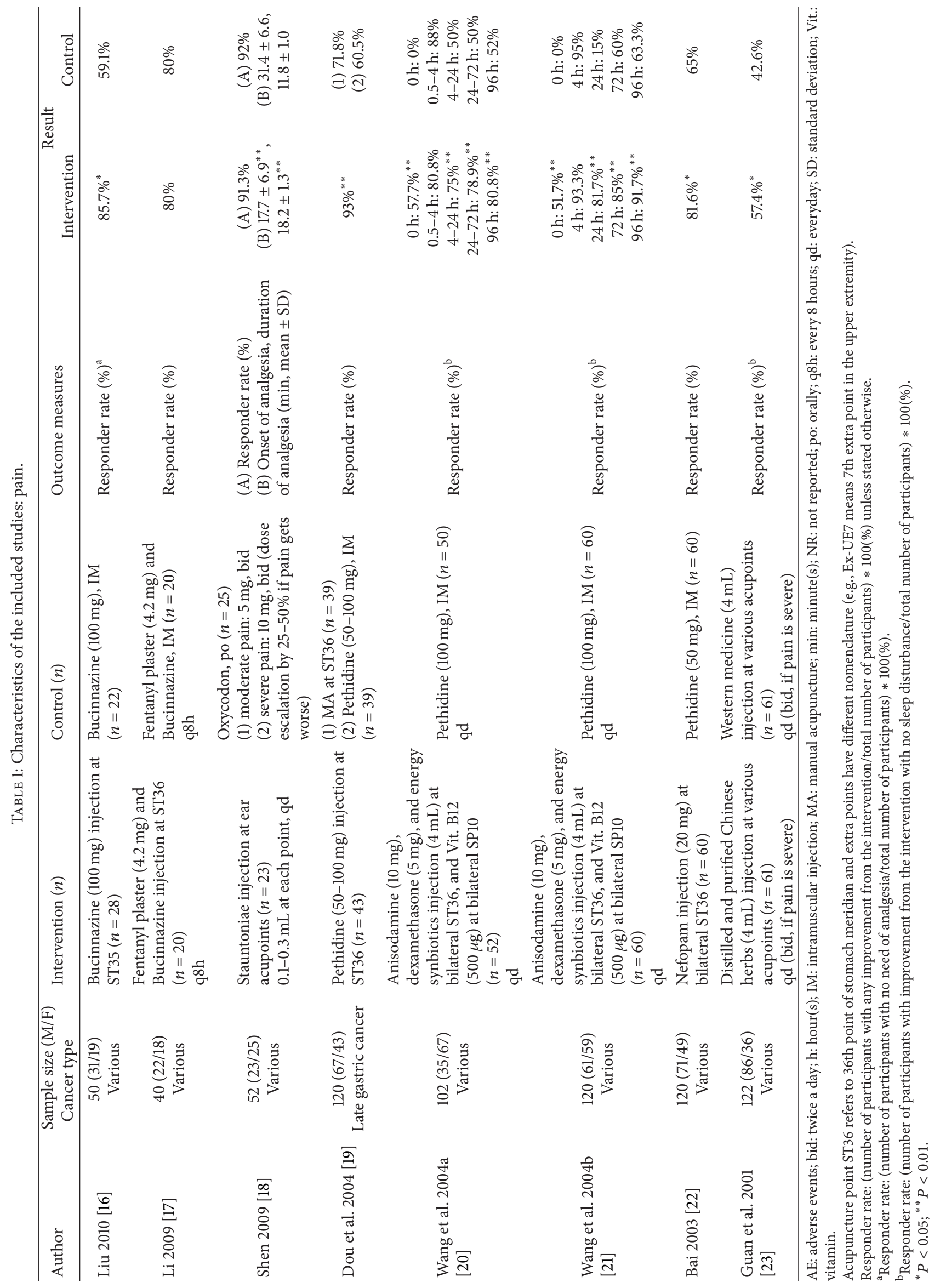




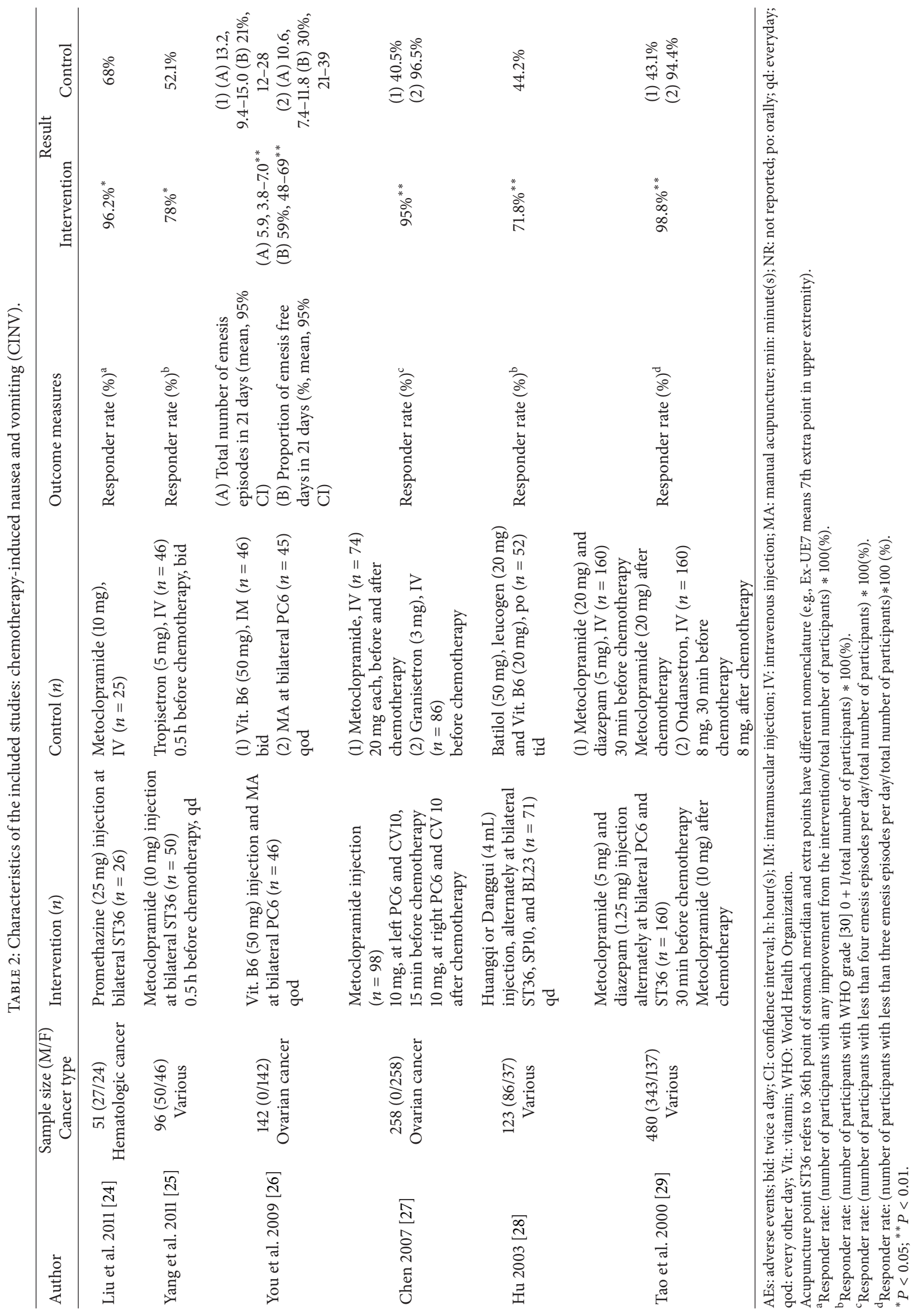




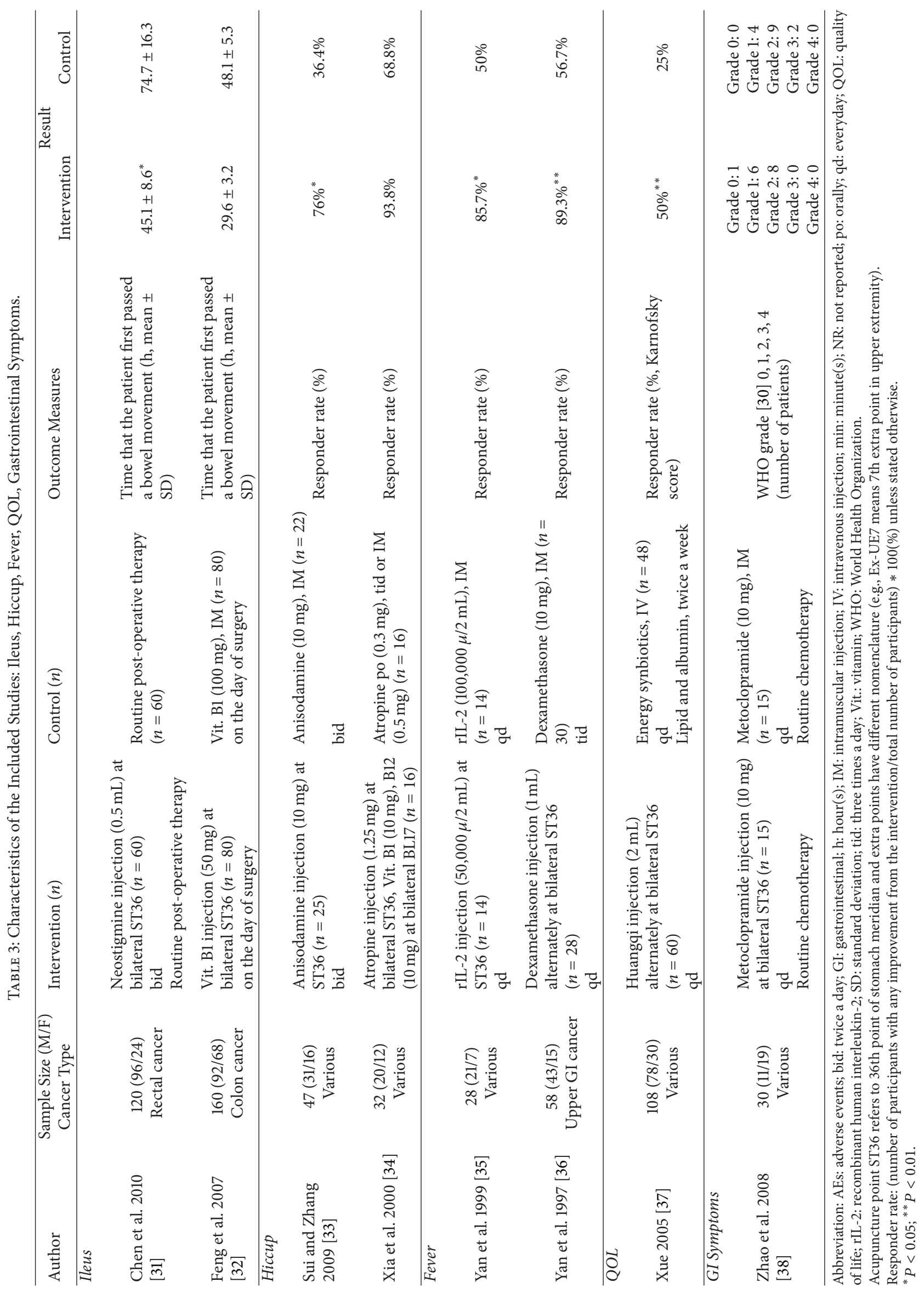


study), and pharmacopuncture with routine chemotherapy versus IV injection with routine chemotherapy.

3.2. ROB Assessment. All of the included articles had a high $\mathrm{ROB}$, lacking proper blinding measures for participants, personnel, and outcome assessors which were participants themselves in all the cases. Only one study reported how they concealed allocation by using sequentially numbered opaque envelopes [26], and none of the other studies reported either how sequence was generated or how concealment of allocation was done so the ROB was evaluated to be unclear. Twenty-one studies had a low ROB for incomplete outcome data and selective outcome reporting categories, but one study [36] had a high ROB for the latter because it reported positive results of symptoms that were not predefined in its method (Table 4).

\subsection{Outcomes of the Included Studies by Symptom}

3.3.1. Pain. All 8 studies used responder rate to evaluate pain (Table 1). They were qualitatively analyzed due to clinical and statistical heterogeneity. Responder rates in 4 studies [17-19, 22] were calculated by percentage of number of participants with any improvement out of total number of participants. Shen [18] also looked at onset time and duration of analgesia in addition to responder rate. In other three studies [20, 21, $23]$, responder rate was defined as the proportion of number of participants with improvement from the intervention and no sleep disturbance. One study [16] used percentage of number of participants with improvement and no need of analgesia out of total number of participants.

In these 8 studies, only one study [17] reported that there was no significant difference of responder rate between pharmacopuncture and control group, and 7 others favored pharmacopuncture. Among the latter 7 studies, three studies had only one control group with one outcome measure, and their details and results are as follows. Liu [16] compared $100 \mathrm{mg}$ of bucinnazine pharmacopuncture at ST36 with bucinnazine IM injection. De-qi was elicited by needle lifting/inserting and twisting. Bai [22] injected $20 \mathrm{mg}$ of nefopam to ST36 and compared responder rate with that of $50 \mathrm{mg}$ pethidine IM injection. Guan et al. [23] compared distilled and purified Chinese herb (chansu, naoyanghua, chuanwu, banxia, xionghuang, and bingpian) pharmacopuncture with western medicine. Acupoints for pharmacopuncture were selected according to each participant's cancer type; for example, participants with lung cancer received pharmacopuncture at LU1, LU2, BL13, and BL17. The above three studies' results showed that pharmacopuncture performs significantly $(P<$ 0.05 ) better than the control.

The other 4 studies had more complicated design than the previous three studies, that is, more than one control group, outcome measure, or outcome measurement time. One study [19] that had two control groups reported that pethidine injection at acupoint ST36 had significantly $(P<$ 0.01 ) better analgesic effects than manual acupuncture at ST36 or pethidine IM injection with flexible dosage between 50 and $100 \mathrm{mg}$. Another study [18] that had two outcome measures compared $0.1-0.3 \mathrm{~mL}$ of Stauntoniae injection at ear acupoints with oral intake of 5-15 mg oxycodone according to degree of pain. While there was no significant difference for responder rate between the two groups $(P>0.05)$, the pharmacopuncture group demonstrated faster onset time (mean $\pm \mathrm{SD}, 17.7 \pm 6.9$ min versus $31.4 \pm 6.6 \mathrm{~min} ; P<0.01$ ) and longer duration $(18.2 \pm 1.3 \mathrm{~min}$ versus $11.8 \pm 1.0 \mathrm{~min} ; P<$ 0.01 ) of analgesia compared to control group. In the study of Wang et al. [20], outcome was measured at more than one time-point. In the pharmacopuncture group, anisodamine hydrochloride $10 \mathrm{mg}$, dexamethasone $5 \mathrm{mg}$, and energy synbiotics $4 \mathrm{~mL}$ were injected at bilateral ST36, and $500 \mu \mathrm{g}$ of vitamin B12 was injected at bilateral SP10. The control group received IM injection of $100 \mathrm{mg}$ pethidine. Injections were given once a day for both groups, and participants were asked about the pain right after the injection, 30 minutes after the injection, and 2, 4, 6 hours after the injection, and then every two hours afterward except sleeping time. No significant difference $(P>0.05)$ was detected when they compared the results of two groups for 30 minutes to 4 hours. The other study [21] by the same authors was done upon a different group of participants, and intervention group and control group were identical. However, this study checked outcome measures 5 times: right after the injection and 4, 24,72 , and 96 hours later. Participants on pharmacopuncture treatment benefited at all time-points except at 4 hours after injection.

In the one study [17], pharmacopuncture group received bucinnazine injection at acupoint ST36, and the control group had IM injection, while $4.2 \mathrm{mg}$ of fentanyl patch was given to both groups. There was no significant difference in the responder rate between the two groups.

3.3.2. CINV. Six trials involving 1,150 participants tested pharmacopuncture with usual care for CINV. All studies favored pharmacopuncture over the control group, but outcome measures varied. Five out of six studies reported responder rate as an outcome measure. One study [24] used responder rate that was calculated by percentage of number of participants with any improvement from the intervention out of total number of participants. Two studies [25, 28] defined the participants with the WHO grades 0 and 1 as responder [30]. Two studies [27, 29] calculated responder rate using emesis episodes: number of participants with less than 4 emesis episodes per day [27] and less than three emesis episodes per day as a responder [29]. One study [26] used two outcome measures, that is, a total number of emesis episodes in 21 days and a proportion of emesis-free days in the same period.

You et al.s study [26] with participants with ovarian cancer tested injection of $50 \mathrm{mg}$ of vitamin B6 either at bilateral PC6 or intramuscularly. There was also a third group and they were treated with manual acupuncture at bilateral PC6. Pharmacopuncture and manual acupuncture were given once every other day with de-qi being elicited. In the control group, IM injection was given twice daily. Total number of emesis episodes and proportion of emesis-free days over 21 days were compared among three groups, and 
TABLE 4: ROB assessment for the included studies by symptom: pain, nausea and vomiting, ileus, hiccup, fever, QOL, and gastrointestinal symptoms.

\begin{tabular}{|c|c|c|c|c|c|c|}
\hline Author & $\begin{array}{c}\text { Random sequence } \\
\text { generation }\end{array}$ & $\begin{array}{c}\text { Allocation } \\
\text { concealment }\end{array}$ & $\begin{array}{l}\text { Blinding of } \\
\text { participants }\end{array}$ & $\begin{array}{c}\text { Blinding of outcome } \\
\text { assessment }\end{array}$ & $\begin{array}{c}\text { Incomplete } \\
\text { outcome data }\end{array}$ & $\begin{array}{l}\text { Selective } \\
\text { reporting }\end{array}$ \\
\hline \multicolumn{7}{|l|}{ Pain } \\
\hline Liu $2010[16]$ & $\mathrm{U}$ & $\mathrm{U}$ & $\mathrm{N}$ & $\mathrm{N}$ & $\mathrm{Y}$ & $\mathrm{Y}$ \\
\hline Li 2009 [17] & $\mathrm{U}$ & $\mathrm{U}$ & $\mathrm{N}$ & $\mathrm{N}$ & $\mathrm{Y}$ & $\mathrm{Y}$ \\
\hline Shen 2009 [18] & $\mathrm{U}$ & $\mathrm{U}$ & $\mathrm{N}$ & $\mathrm{N}$ & $\mathrm{Y}$ & $\mathrm{Y}$ \\
\hline Dou et al. 2004 [19] & $\mathrm{U}$ & $\mathrm{U}$ & $\mathrm{N}$ & $\mathrm{N}$ & $\mathrm{Y}$ & $\mathrm{Y}$ \\
\hline Wang et al. 2004a [20] & $\mathrm{U}$ & $\mathrm{U}$ & $\mathrm{N}$ & $\mathrm{N}$ & $\mathrm{Y}$ & $\mathrm{Y}$ \\
\hline Wang et al. 2004b [21] & $\mathrm{U}$ & $\mathrm{U}$ & $\mathrm{N}$ & $\mathrm{N}$ & $\mathrm{Y}$ & $\mathrm{Y}$ \\
\hline Bai 2003 [22] & $\mathrm{U}$ & $\mathrm{U}$ & $\mathrm{N}$ & $\mathrm{N}$ & $\mathrm{Y}$ & $\mathrm{Y}$ \\
\hline Guan et al. 2001 [23] & $\mathrm{U}$ & $\mathrm{U}$ & $\mathrm{N}$ & $\mathrm{N}$ & $\mathrm{Y}$ & $\mathrm{Y}$ \\
\hline \multicolumn{7}{|l|}{ Nausea and vomiting } \\
\hline Liu et al. 2011 [24] & $\mathrm{U}$ & $\mathrm{U}$ & $\mathrm{N}$ & $\mathrm{N}$ & $\mathrm{Y}$ & $\mathrm{Y}$ \\
\hline Yang et al. 2011 [25] & $\mathrm{U}$ & $\mathrm{U}$ & $\mathrm{N}$ & $\mathrm{N}$ & $\mathrm{Y}$ & $\mathrm{Y}$ \\
\hline You et al. 2009 [26] & $\mathrm{U}$ & $\mathrm{Y}$ & $\mathrm{N}$ & $\mathrm{N}$ & $\mathrm{Y}$ & $\mathrm{Y}$ \\
\hline Chen 2007 [27] & $\mathrm{U}$ & $\mathrm{U}$ & $\mathrm{N}$ & $\mathrm{N}$ & $\mathrm{Y}$ & $\mathrm{Y}$ \\
\hline Hu 2003 [28] & $\mathrm{U}$ & $\mathrm{U}$ & $\mathrm{N}$ & $\mathrm{N}$ & $\mathrm{Y}$ & $\mathrm{Y}$ \\
\hline Tao et al. 2000 [29] & $\mathrm{U}$ & $\mathrm{U}$ & $\mathrm{N}$ & $\mathrm{N}$ & $\mathrm{Y}$ & $\mathrm{Y}$ \\
\hline \multicolumn{7}{|l|}{ Ileus } \\
\hline Chen et al. 2010 [31] & $\mathrm{U}$ & $\mathrm{U}$ & $\mathrm{N}$ & $\mathrm{N}$ & $\mathrm{Y}$ & $\mathrm{Y}$ \\
\hline Feng et al. 2007 [32] & $\mathrm{U}$ & $\mathrm{U}$ & $\mathrm{N}$ & $\mathrm{N}$ & $\mathrm{Y}$ & $\mathrm{Y}$ \\
\hline \multicolumn{7}{|l|}{ Hiccup } \\
\hline Sui and Zhang 2009 [33] & $\mathrm{U}$ & $\mathrm{U}$ & $\mathrm{N}$ & $\mathrm{N}$ & Y & $\mathrm{Y}$ \\
\hline Xia et al. 2000 [34] & $\mathrm{U}$ & $\mathrm{U}$ & $\mathrm{N}$ & $\mathrm{N}$ & $\mathrm{Y}$ & $\mathrm{Y}$ \\
\hline \multicolumn{7}{|l|}{ Fever } \\
\hline Yan et al. 1999 [35] & $\mathrm{U}$ & $\mathrm{U}$ & $\mathrm{N}$ & $\mathrm{N}$ & $\mathrm{Y}$ & $\mathrm{Y}$ \\
\hline Yan et al. 1997 [36] & $\mathrm{U}$ & $\mathrm{U}$ & $\mathrm{N}$ & $\mathrm{N}$ & $\mathrm{Y}$ & $\mathrm{N}$ \\
\hline \multicolumn{7}{|l|}{ QOL } \\
\hline Xue 2005 [37] & $\mathrm{U}$ & $\mathrm{U}$ & $\mathrm{N}$ & $\mathrm{N}$ & $\mathrm{Y}$ & $\mathrm{Y}$ \\
\hline \multicolumn{7}{|l|}{ Gastrointestinal symptoms } \\
\hline Zhao et al. 2008 [38] & $\mathrm{U}$ & $\mathrm{U}$ & $\mathrm{N}$ & $\mathrm{N}$ & $\mathrm{Y}$ & $\mathrm{Y}$ \\
\hline
\end{tabular}

ROB was assessed using the ROB assessment tool from the Cochrane Handbook for Systematic Reviews of Interventions [39].

$\mathrm{N}$ : no (high risk of bias); QOL: quality of life; ROB: risk of bias; U: Unclear (uncertain risk of bias); Y: yes (low risk of bias).

the pharmacopuncture group significantly reduced emesis compared to both control groups $(P<0.01)$.

Among remaining 5 studies, three studies' responder rates were determined by severity of symptoms; Liu et al. [24] used $25 \mathrm{mg}$ of promethazine for injection at bilateral ST36 for pharmacopuncture group. For control group, they used $10 \mathrm{mg}$ metoclopramide IV injection. Pharmacopuncture group's responder rate was significantly higher than that of control group ( $96.2 \%$ versus $68 \%$; $P<0.05$ ). Yang et al. [25] also reported that significantly more participants in the pharmacopuncture group responded to the treatment than the control group ( $78 \%$ versus $52.1 \%$; $P<0.05$ ). The pharmacopuncture group was given $10 \mathrm{mg}$ of metoclopramide at bilateral ST36 once daily. On the other hand, $5 \mathrm{mg}$ of tropisetron IV injection was given to control group twice daily. $\mathrm{Hu}$ [28] injected $4 \mathrm{~mL}$ of Chinese herbs (huangqi or danggui) once a day for pharmacopuncture group at 5 acupoints: bilateral ST36, bilateral SP10, and BL23. Control group had oral medication of $50 \mathrm{mg}$ of batilol, $20 \mathrm{mg}$ of leucogen, and $20 \mathrm{mg}$ vitamin B6, three times a day. Reported responder rate indicated that pharmacopuncture group had significantly $(P<0.01)$ better outcome than control group. Pooling these three studies yielded a significant benefit of pharmacopuncture against control group in symptom improvement of CINV (RR $1.28,95 \%$ CI $(1.14,1.44), \chi^{2}=0.65, \mathrm{df}=2, P=0.72 ; I^{2}=0 \%$; Figure 2(a)).

In the other two studies, number of emetic episodes was used to calculate responder rate. Chen [27] compared metoclopramide injections at acupoints with two control groups where IV injection of metoclopramide and IV injection of granisetron were given; pharmacopuncture group had $10 \mathrm{mg}$ of injection for left PC6 and CV10 each, 15 minutes before 


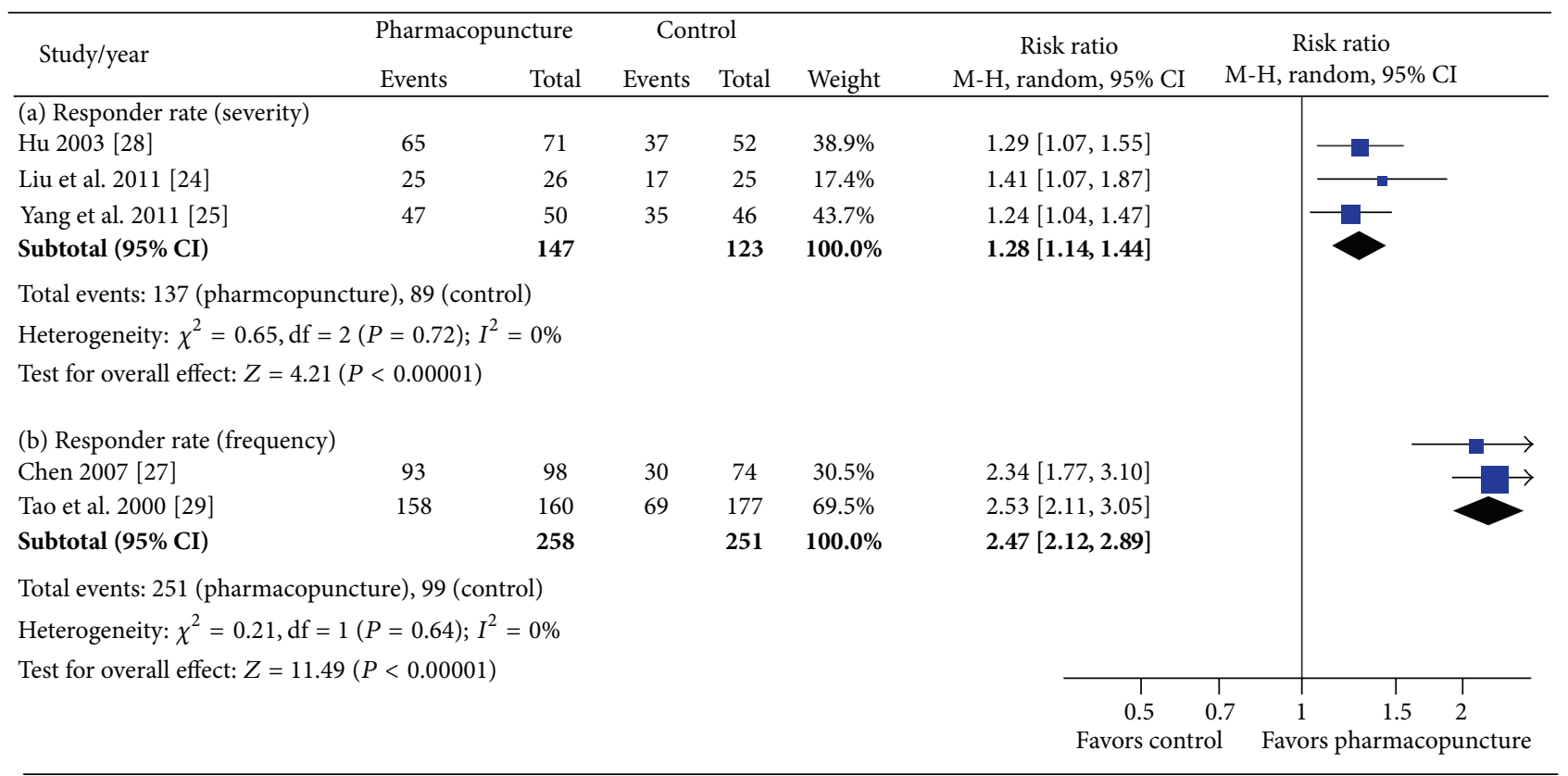

FIGURE 2: Forest plot of the effect of pharmacopuncture for CINV. CINV: chemotherapy-induced nausea and vomiting.

chemotherapy, and then had another $10 \mathrm{mg}$ each at right PC6 and CV10 right after chemotherapy. Metoclopramide control group had $20 \mathrm{mg}$ injection each 15 minutes before and after chemotherapy, and granisetron group had $3 \mathrm{mg}$ injection before chemotherapy. Results showed that pharmacopuncture had a higher responder rate than metoclopramide control group (95\% versus 40.5\%; $P<0.01$ ), and no difference emerged when compared with granisetron group (95\% versus $96.5 \%$; $P>0.05$ ). Tao et al. [29] also compared three groups. Pharmacopuncture group had $5 \mathrm{mg}$ of metoclopramide and $1.25 \mathrm{mg}$ of diazepam injection at bilateral PC6 and ST36, 30 minutes before chemotherapy, then $10 \mathrm{mg}$ of metoclopramide right after chemotherapy. In a similar way, one control group had $20 \mathrm{mg}$ of metoclopramide and $5 \mathrm{mg}$ of diazepam and then $20 \mathrm{mg}$ of metoclopramide, IV injection. The other group was given $8 \mathrm{mg}$ of IV injection of ondansetron 30 minutes before and right after chemotherapy. Responder rate was reported for each group and pharmacopuncture group had better results than metoclopramide control group $(98.8 \%$ versus 43.1\%; $P<0.01$ ) and no different result when compared with ondansetron group (98.8\% versus $94.4 \% ; P>0.05)$. Combining these two studies showed that pharmacopuncture significantly reduced frequency of CINV compared with usual care (RR 2.47, 95\% CI $(2.12,2.89), \chi^{2}=0.21$, df $=1$, $P=0.64 ; I^{2}=0 \%$; Figure $\left.2(\mathrm{~b})\right)$.

3.3.3. Ileus, Hiccup, Fever, QOL, and GI Symptoms. Of the 8 studies included, 5 studies of fever $[35,36]$, hiccup $[33,34]$, and QOL [37] reported responder rate, that is, number of participants with any improvement from the intervention out of total number of participants (\%). However, Xue [37] mentioned that they used Karnofsky score to assess improvement. Two studies [31,32] of postoperative ileus measured time that the patient first passed a bowel movement. The last study [38] used WHO grades [30] to evaluate GI symptoms.

Chen et al. [31] and Feng et al. [32] studied effectiveness of pharmacopuncture for postoperative recovery of colorectal cancer patients. Rectal cancer patients participated in the study of Chen et al. [31] and the study reported that pharmacopuncture group's time that the patient first passed a bowel movement was significantly shorter than that of control group $(45.1 \pm 8.64$ hours $(\mathrm{h})$ versus $74.7 \pm 16.32 \mathrm{~h}$; $P<0.05)$. Pharmacopuncture group received injection of neostigmine at bilateral ST36, $0.5 \mathrm{~mL}$ each in addition to routine postoperative care twice daily, while the control group was given routine postoperative care alone. In Feng et al. study [32], colon cancer patients were recruited. Time that the patient first passed a bowel movement in the pharmacopuncture group was significantly shorter than that of the control group $(29.6 \pm 3.2 \mathrm{~h}$ versus $48.1 \pm 5.3 \mathrm{~h} ; P<$ 0.01). Both groups received $100 \mathrm{mg}$ of vitamin B1 injection, but intervention group's injection was at bilateral ST36 with de-qi being elicited, and control group received IM injection.

Two studies $[33,34]$ had participants with hiccup. Sui and Zhang [33] reported that pharmacopuncture group had significantly higher responder rate than control group $(P<0.05)$. Intervention group was injected with $10 \mathrm{mg}$ of anisodamine at ST36 twice a day. Control group got $10 \mathrm{mg}$ of anisodamine injected intramuscularly twice a day. Xia et al. [34] also reported that intervention group had higher responder rate than control group, but the difference was not significant $(P>0.05)$. They injected $1.25 \mathrm{mg}$ of atropine at bilateral ST36 and $10 \mathrm{mg}$ each of vitamin B1 and B12 at bilateral BL17 for pharmacopuncture group. Control group had either oral intake of $0.3 \mathrm{mg}$ of atropine three times a day, or $0.5 \mathrm{mg}$ IM injection of atropine. 
Two studies $[35,36]$ of fever drew a conclusion that pharmacopuncture group had significantly better result compared to control group $(P<0.05)$. In Yan et al. study [35], the intervention group had pharmacopuncture treatment with $50,000 \mu / 2 \mathrm{~mL}$ of recombinant human interleukin-2 (rIL-2) at ST36 once daily. For control group, rIL-2 $(100,000 \mu / 2 \mathrm{~mL})$ IV was administered once daily. Another study by the same author [36] used dexamethasone to compare pharmacopuncture and IM injection; pharmacopuncture group got $1 \mathrm{~mL}$ of dexamethasone at bilateral ST36 alternately once a day and de-qi was elicited before the injection. The control group had $10 \mathrm{mg}$ of IM injection three times a day. Treatments continued for 5 days.

Xue [37] looked at participants' QOL and used Karnofsky score to calculate responder rate of the two groups. Pharmacopuncture significantly improved QOL compared to control group $(P<0.01)$. Intervention group had Huangqi injection at bilateral ST36 and the injection was once a day, alternately, with a dose of $2 \mathrm{~mL}$. Control group received synbiotics IV injection once a day, and also lipid and albumin were given twice weekly. Treatment spanned two weeks.

Lastly, Zhao et al. [38] investigated the effect of metoclopramide pharmacopuncture on GI symptoms, that is, nausea, vomiting, diarrhea, and xerostomia. They reported that intervention group and control group did not have significantly different results $(P>0.05)$. In addition to routine chemotherapy, the intervention group received $10 \mathrm{mg}$ injection at bilateral ST36 once a day, and the control group had IM injection. They assessed the improvement of GI symptoms on WHO 5 grades [30] starting from 0 to 5 , grade 0 being the best outcome. From pharmacopuncture group, one participant matched criteria for grade 0 . Then there were 6,8 , 0 , and 0 participants for grades $1,2,3$, and 4 . In control group, none of the participants were suitable for grade 0 , and 4 were in grade 1 . Then there were 9,2 , and 0 participants for grades 2,3 , and 4 .

3.4. Safety Evaluation. Of the 22 studies, $50 \%$ of the studies did not mention AEs at all, and the other half described AEs or mentioned that there were no AEs reported by the participants (Table 5). Five studies out of 11 reported that there were no AEs in the intervention group.

In the 6 studies that reported specific AEs for both groups, all mentioned that AEs were resolved after the end of treatment or spontaneously. However, none of the studies clarified causal relationship of AEs and pharmacopuncture. Six studies could be categorized into three groups: identical drug used for both intervention and control groups $[16,33$, $36]$, different drugs used for each group [20, 21], and one study [29] with two control groups which correspond to identical drug and different drug each. While the other four studies provided numerical data by each group, two studies $[20,21]$ lacked such information and just listed patientreported AEs.

Among the five studies that reported numerical data for intervention and control group, the incidence rate ranged from $0 \%$ to $36.0 \%$ for intervention group $[18,33]$ and $33.3 \%$ to $72.0 \%$ for control group $[18,36]$. No dropout or withdrawal was reported in any of the studies.

\section{Discussion}

In this review, qualitative synthesis and partial meta-analysis regarding effectiveness of pharmacopuncture for cancerrelated symptoms were done. Twenty-two included studies involving 2,459 participants were classified under 7 categories and for studies of CINV, a meta-analysis was performed. Pharmacopuncture significantly reduced severity and frequency of CINV compared with usual care. For studies that were under categories other than CINV, various study design and methods led us to qualitative synthesis of evidence. Among 8 studies under pain category, one study [17] reported a result indicating no difference between pharmacopuncture and conventional IM analgesic injection, but others were mostly in favor of pharmacopuncture against conventional treatments. In studies related to ileus, hiccup, fever, QOL, and GI symptoms, reported figures for results varied, but overall, all results were favoring pharmacopuncture. Fifty percent $(11 / 22)$ of the included studies reported occurrence or absence of AEs, and among the reported AEs, there were no serious AEs that led to withdrawals from the study. However, we argue that the overall estimated results from either qualitative synthesis or meta-analysis should be interpreted with caution because of included studies' innate limitations, especially considering the fact that all included studies suffered from a high ROB and clinical heterogeneity.

We used Cochrane ROB criteria [39] for assessing ROB for the included studies. Overall ROB was high for the included studies; all failed to address the random sequence generation method in detail, and also all studies had unclear risk for allocation concealment except one [26] that mentioned usage of central randomization and sequentially numbered opaque envelopes. Participant blinding in pharmacopuncture studies may be more easily achievable than other nonpharmacological interventions such as acupuncture because pharmacopuncture is mostly compared against IV or IM injection. Nevertheless, none of the participants, personnel, and outcome assessors were blinded in the included studies and this could have brought performance and/or detection bias. High ROB of the primary studies in this field has been one of the major challenges for CAM to establish its place in evidence-based cancer care $[42,43]$. Our review is no exception; the limitation of present study mainly lies on the high ROB of the included studies which was criticized above.

Another limitation of this review is from the clinical heterogeneity of the included studies. Study participants were often of different types and stages of cancer, causes of complained symptoms were not specified, duration of intervention and follow up length after the intervention were missing in some studies, and in many cases some of the selected control groups were not the best evidencebased treatment available [44, 45]. As with acupuncture, pharmacopuncture intervention varied greatly across trials that estimating its effectiveness under the single term of pharmacopuncture may be practically difficult. All these 
TABLE 5: Reported AEs in the included studies.

\begin{tabular}{|c|c|c|c|}
\hline $\begin{array}{l}\text { Author } \\
\text { Sample Size* (Int./Cont.) }\end{array}$ & Condition & Intervention group & Control group \\
\hline \multicolumn{4}{|c|}{ Reported “No AEs” for intervention group } \\
\hline Liu et al. $2011[24]$ & CINV & No AEs occurred & No AEs occurred \\
\hline Yang et al. 2011 [25] & CINV & No AEs occurred & NR \\
\hline Chen 2007 [27] & CINV & No AEs occurred & NR \\
\hline Xue 2005 [37] & QOL & No AEs occurred & NR \\
\hline $\begin{array}{l}\text { Shen } 2009[18] \\
23 / 25\end{array}$ & Pain & No AEs occurred & $\begin{array}{l}\text { Constipation (9), nausea and vomiting (6), } \\
\text { dizziness (3) }\end{array}$ \\
\hline \multicolumn{4}{|c|}{ Identical Drug Used for Both Groups } \\
\hline $\begin{array}{l}\text { Liu } 2010[16] \\
28 / 19\end{array}$ & Pain & $\begin{array}{l}\text { Nausea and vomiting (1), headache and } \\
\text { dizziness ( } 2 \text { ) } \\
\text { Resolved after the end of treatment }\end{array}$ & $\begin{array}{l}\text { Nausea and vomiting (3), headache and } \\
\text { dizziness (5), drowsiness and fatigue ( } 2) \\
\text { Resolved after the end of treatment }\end{array}$ \\
\hline $\begin{array}{l}\text { Sui and Zhang } 2009[33] \\
25 / 22\end{array}$ & Hiccup & $\begin{array}{l}\text { Xerostomia (9) } \\
\text { Resolved after the end of treatment }\end{array}$ & $\begin{array}{l}\text { Xerostomia (10) } \\
\text { Resolved after the end of treatment }\end{array}$ \\
\hline $\begin{array}{l}\text { Tao et al. } 2000[29]^{* *} \\
160 / 160\end{array}$ & CINV & $\begin{array}{l}\text { Headache and dizziness (26), bloating and } \\
\text { constipation (4), and diarrhea (6) } \\
\text { Resolved after the end of treatment }\end{array}$ & $\begin{array}{l}\text { Headache and dizziness (33), bloating and } \\
\text { constipation ( } 9 \text { ), and diarrhea (20) } \\
\text { Resolved after the end of treatment }\end{array}$ \\
\hline $\begin{array}{l}\text { Yan et al. } 1997[36] \\
28 / 30\end{array}$ & Fever & $\begin{array}{l}\text { Local pain and limitation of lower limb } \\
\text { movement (5) } \\
\text { Resolved after the end of treatment }\end{array}$ & $\begin{array}{l}\text { Insomnia and increased talkativeness (8) } \\
\text { and panhidrosis }(2) \\
\text { Did not affect further treatment }\end{array}$ \\
\hline \multicolumn{4}{|c|}{ Different Drugs Used for Each Groups } \\
\hline $\begin{array}{l}\text { Wang et al. } 2004 \mathrm{a}^{* * *} \\
\text { [20] }\end{array}$ & Pain & $\begin{array}{l}\text { Xerostomia, hot flashes, and blurred vision } \\
\text { Relieved spontaneously }\end{array}$ & $\begin{array}{l}\text { Nausea, vomiting, constipation, and urinary } \\
\text { retention } \\
\text { Mild and tolerable level }\end{array}$ \\
\hline $\begin{array}{l}\text { Wang et al. } 2004 b^{* * *} \\
{[21]}\end{array}$ & Pain & $\begin{array}{l}\text { Xerostomia, hot flashes, and blurred vision } \\
\text { Relieved spontaneously } \\
\text { Temporary low blood sugar } \\
\text { Continued treatment after glucose IV } \\
\text { injection or a meal }\end{array}$ & $\begin{array}{l}\text { Nausea, vomiting, constipation, and urinary } \\
\text { retention } \\
\text { Mild and tolerable level }\end{array}$ \\
\hline $\begin{array}{l}\text { Tao et al. } 2000[29]^{* *} \\
160 / 160\end{array}$ & CINV & $\begin{array}{l}\text { Headache and dizziness (26), bloating and } \\
\text { constipation (4), and diarrhea (6) } \\
\text { Resolved after the end of treatment }\end{array}$ & $\begin{array}{l}\text { Headache and dizziness (29), bloating and } \\
\text { constipation (22), and diarrhea (5) } \\
\text { Resolved after the end of treatment }\end{array}$ \\
\hline \multicolumn{4}{|c|}{$\begin{array}{l}\text { AEs: adverse events; CINV: chemotherapy-induced nausea and vomiting; Cont.: control group; Int.: intervention group; IV: intravenous; NR: not reported; } \\
\text { QOL: quality of life. } \\
\text { Number in the parenthesis is reported number of cases. } \\
{ }^{*} \text { Sample size is reported on this table for studies with numerical data only. } \\
{ }^{* *} \text { Two different control groups yielded two separate documentations in this table. } \\
{ }^{* * *} \text { Number of cases for symptoms were not reported. }\end{array}$} \\
\hline
\end{tabular}

resulted in clinical heterogeneity of the included studies, which precluded us from pooling studies or drawing a definitive conclusion. Therefore, our findings should be interpreted with consideration of these limitations.

In RCTs, reporting harms as well as its benefits for an intervention is crucial to achieve a balanced perspective on the intervention. According to a guideline for better reporting of harms in RCTs, not only description of AEs for each arm should be provided, but also more rigor explanations like definitions for AEs or how harms-related information was collected should be reported [46]. In this review, only half of the studies briefly reported information regarding AEs, that is, just listing the symptoms, and no causality nor any further explanation was given. There were 5 studies reporting that there were no AEs, but this should be read cautiously because an intervention cannot be labeled safe merely based on the absence of information [47]. Interestingly, of the 4 studies that used identical drug for both groups, three studies' reported AEs from intervention group totally overlapped with those of control group. For example, Sui and Zhang [33] reported that there were a number of participants with xerostomia for both groups injected with anisodamine. Also, two other studies $[20,21]$ that administered anisodamine for their pharmacopuncture group reported AEs such as xerostomia along with hot flashes and blurred vision, which are all commonly known AEs of anisodamine itself [48, 49]. This can be suggesting that those AEs may be caused by the drug itself and not by the modality of pharmacopuncture. Still, more convincing evidence is needed to accurately assess the safety of pharmacopuncture.

One of the strengths of this systematic review is that we conducted comprehensive electronic database search and 
hand search for articles written in English, Chinese, and Korean. However, we cannot rule out that we might have missed some relevant articles. Moreover, all the included studies were from China at the end, so generalization of this review's finding is somewhat limited. Nonetheless, pharmacopuncture is evidently gaining popularity $[8,50]$, yet there was no other systematic review assessing pharmacopuncture's efficacy for various cancer-related symptoms. Therefore, this review serves as the first summarized evidence of its kind and may provide basis for further studies.

Pharmacopuncture has served as a novel and useful treatment for various diseases, usually in China and Korea $[8,50]$. Given that most of its usage and relevant studies are from locally limited area, some consideration may be required before it is used worldwide, but the amount of data is increasing continuously [50] and so far the results are promising [51, 52]. For instance, a case series of bee venom pharmacopuncture for chemotherapy-induced peripheral neuropathy reported improvement of symptoms in patients and, importantly, there were no related AEs [52]. There was also a noticeable pilot study from University of California, San Francisco, that tested pharmacopuncture for primary dysmenorrhea, and they suggested that pharmacopuncture is highly acceptable among American women as well [51].

With a growing interest and increasing use of CAM in general, cancer patients are even more interested in various modalities of CAM, and they usually view it in a positive way with high levels of satisfaction $[6,53]$. In this context, pharmacopuncture's utility for cancer-related symptoms is worth noticing. Especially, a brief Chinese review article of pharmacopuncture for cancer-related symptoms concluded that pharmacopuncture is not only effective but also costeffective [54]. However, positive findings of that review can be consequences of publication bias and/or other methodological flaws. Also, the review itself suffers from poor reporting such as lacking proper explanation of which databases were searched with what kind of inclusion criteria. Though our review has a similar limitation, the execution of structured assessment and synthesis of up-to-date evidence may have been better delivered in our review to provide the best evidence possible.

This review suggests that the current evidence on pharmacopuncture for cancer-related symptoms is not sufficient yet. Included studies do report favorable results, but limitations from their quality make it hard to fully substantiate pharmacopuncture's effectiveness. To come up with a strong level of evidence, future studies should work on improving their quality and reporting. For instance, reporting part of the study can improve and become more reliable just by following existing guidelines. The Consolidated Standards of Reporting Trials (CONSORT) statement [55] is a well-recognized tool researchers can refer to. For trials reporting results of cancer treatment, there is also a classic guideline specifically for that purpose [56]. Referring to such guideline will improve quality of a study by giving detailed information about participants' status. Also, since practice of pharmacopuncture is very similar to acupuncture treatment in many steps, applying the Standards for Reporting Interventions in Clinical Trials of Acupuncture (STRICTA) [57] with suitable modification will be helpful as well. For example, 18 studies included in this review injected medication at ST36 but did not clarify why it was chosen. There was an interesting study from China that compared responder rate of injecting metoclopramide at different acupoints for CINV, and they reported that ST36 group showed significantly better result compared to LI4 and PC6 group (95.3\% versus 79.1\%, $P<0.05$ ) [58]. Providing rationale or relevant evidence for chosen acupoints will improve the completeness and transparency of reporting in a study and add accuracy for future interpretation and replication of the study [57]. In this review, only handful of studies were subject to a meta-analysis due to high heterogeneity. When there are enough number of highquality studies for each symptom, strength of evidence for pharmacopuncture may increase, and it will contribute to improvement of provided care for cancer patients.

\section{Conclusion}

We conclude that the level of evidence is not strong enough to draw any firm conclusion and gives us only a cautious suggestion that pharmacopuncture may help alleviate cancerrelated pain, CINV, and other various symptoms such as ileus, hiccup, fever, QOL, and GI symptoms. To confirm this finding, further rigorously designed and conducted studies are required.

\section{Conflict of Interests}

All authors declare that there is no conflict of interests regarding the publication of this paper.

\section{Authors' Contribution}

Soyeon Cheon, Seung-Hun Cho, and Hyangsook Lee were responsible for the study concept and design; Soyeon Cheon, In-Seon Lee, and Xiuyu Zhang were responsible for literature searching; Soyeon Cheon, Younbyoung Chae, and Hyangsook Lee were responsible for data analysis and interpretation; Soyeon Cheon, In-Seon Lee, and Hyangsook Lee drafted the paper; Hyangsook Lee supervised the study; all authors participated in the analysis and interpretation of data and approved the final paper.

\section{Acknowledgments}

This work was supported by the National Research Foundation of Korea (NRF) Grant funded by the Korean government (Ministry of Science, ICT \& Future Planning) (no. 2013R1A6A6029251) and a Grant from the National R \& D Program for Cancer Control, Ministry for Health \& Welfare, Republic of Korea (1020330).

\section{References}

[1] A. Jemal, F. Bray, M. M. Center, J. Ferlay, E. Ward, and D. Forman, "Global cancer statistics," CA Cancer Journal for Clinicians, vol. 61, no. 2, pp. 69-90, 2011. 
[2] R. Siegel, D. Naishadham, and A. Jemal, "Cancer statistics, 2013," CA Cancer Journal for Clinicians, vol. 63, no. 1, pp. 11-30, 2013.

[3] B. Stewart and C. P. Wild, World Cancer Report 2014, International Agency for Research on Cancer, Lyon, France, 2014.

[4] T. Gansler, P. A. Ganz, M. Grant et al., "Sixty years of CA: a cancer journal for clinicians," CA Cancer Journal for Clinicians, vol. 60 , no. 6, pp. 345-350, 2010.

[5] J. N. Weitzel, K. R. Blazer, D. J. MacDonald, J. O. Culver, and K. Offit, "Genetics, genomics, and cancer risk assessment: state of the Art and Future Directions in the Era of Personalized Medicine," CA Cancer Journal for Clinicians, vol. 61, no. 5, pp. 327-359, 2011.

[6] J. G. Anderson and A. G. Taylor, "Use of complementary therapies for cancer symptom management: Results of the 2007 National Health Interview Survey," Journal of Alternative and Complementary Medicine, vol. 18, no. 3, pp. 235-241, 2012.

[7] K. Arthur, J. C. Belliard, S. B. Hardin, K. Knecht, C. S. Chen, and S. Montgomery, "Practices, attitudes, and beliefs associated with complementary and alternative medicine (CAM) use among cancer patients," Integrative Cancer Therapies, vol. 11, no. 3, pp. 232-242, 2012.

[8] K. Kwon, S. Kim, C. Kim et al., Pharmacopuncturology, Elsevier, Republic of Korea, Seoul, 2 edition, 2011.

[9] M. W. Strudwick, R. C. Hinks, and S. T. B. Choy, "Point injection as an alternative acupuncture technique-an exploratory study of responses in healthy subjects," Acupuncture in Medicine, vol. 25, no. 4, pp. 166-174, 2007.

[10] H. Song, J. Choi, J. Kang, and H. Lee, "The effect of the acupuncture therapy in combination with soyeom pharmacopuncture therapy on the improvement of the symptoms of the patients with herniated intervertebral disk of L-spine in his initial stage of hospitalization," Journal of Korean Pharmacopuncture Institute, vol. 12, no. 4, pp. 111-118, 2009.

[11] S. Yu, J. Lee, K. Kwon, and H. Lee, "Comparative Study of Acupuncture, Bee Venom Acupuncture, and Bee Venom Pharmacopuncture on the Treatment of Herniation of Nucleus Pulpous," The Journal of Korean Acupuncture \& Moxibustion Society, vol. 23, no. 5, pp. 39-54, 2006.

[12] J. Choi, H. Lee, J. Kang et al., "Comparative Study of General Oriental Medical Treatment and Bee Venom Pharmacopuncture on Acute Peripheral Facial Paralysis Patient with Postauricular Pain," The Journal of Korean Acupuncture \& Moxibustion Society, vol. 26, no. 5, pp. 95-103, 2009.

[13] S.-K. Jung, F.-Y. Shen, and M. S. Lee, "Effectiveness of pharmacopuncture for asthma: a systematic review and meta-analysis," Evidence-based Complementary and Alternative Medicine, vol. 2011, Article ID 678176, 7 pages, 2011.

[14] Z. Yan, N. Ding, and H. Hua, "A systematic review of acupuncture or acupoint injection for management of burning mouth syndrome," Quintessence International, vol. 43, no. 8, pp. 695701, 2012.

[15] J. Liu, L. Ma, M. Han, C. Liu, and X. Pan, "P04. 56. Acupoint versus non-acupoint injection of antiviral treatment for chronic hepatitis B: a systematic review of randomized controlled trials," BMC Complementary and Alternative Medicine, vol. 12, supplement 1, p. 326, 2012.

[16] H. Liu, "Efficacy and care of acupoint injection therapy for abdominal pain of cancer," Journal of Emergency in Traditional Chinese Medicine, vol. 19, no. 3, pp. 527-528, 2010.

[17] S. Li, "Clinical observation of the fentanyl patch and bucinnazine injecation at acupoint zusanli for cancer pain," Chinese Medicine Herald, vol. 6, no. 22, p. 82, 2009.
[18] Q. Shen, Clinical Observation and Mechanism Study on the Treatment of Cancer Pain with Ear Acupoints Injection, Guangzhou University of Chinese Medicine, 2009.

[19] Z. Dou, Y. Lei, G. Zheng, and B. Hong, "Observation on the abirritation of Zusanli point injection on late Gastric Carcinoma," Zhejiang Journal of Integrated Traditional Chinese and Western Medicine, vol. 14, no. 9, pp. 538-539, 2004.

[20] S. Wang, Y. Guo, and X. Zhang, "Observation and care of acupoint injection treatment for abdominal pain caused by radiotherapy for malignant tumor," Chinese Journal of Practical Nursing, vol. 20, no. 7, pp. 1-2, 2004.

[21] S. Wang, X. Zhu, and Y. Guo, "Clinical study of point injection in treatment of abdominal pain caused by malignant tumor chemotherapy," Journal of Zhengzhou University, vol. 39, no. 4, pp. 651-652, 2004.

[22] Y. Bai, "Effect of nefopam acupoint injection for treatment of cancer pain," Traditional Chinese Medicine in Inner Mongolia, vol. 5, p. 33, 2003.

[23] H. Guan, F. Du, and X. Yin, "Analysis of the efficacy of herbal injection for cancer pain," Modern Rehabilitation, vol. 5, no. 11, p. 128, 2001.

[24] Z. Liu, Y. Xiao, Z. Jiang et al., "Effect of promethazine acupoint injection at ST36 on intractable vomiting in patients with hematologic cancer after chemotherapy," Guangdong Medical Journal, vol. 32, no. 13, pp. 1769-1770, 2011.

[25] C. Yang, X. Wang, and Q. Gao, "A randomized controlled trial of prophylaxis of gastrointestinal reaction by acupoint injection at ST36 in patients with cancer after chemotherapy," Henan Traditional Chinese Medicine, vol. 31, no. 8, pp. 890-891, 2011.

[26] Q. You, H. Yu, D. Wu, Y. Zhang, J. Zheng, and C. Peng, "Vitamin B6 points PC6 injection during acupuncture can relieve nausea and vomiting in patients with ovarian cancer," International Journal of Gynecological Cancer, vol. 19, no. 4, pp. 567-571, 2009.

[27] Z. Chen, "A clinical observation of aqua-acupuncture treatment for chemotherapy-induced vomiting in ovarian cancer patients," Hebei Medical Journal, vol. 28, no. 4, p. 351, 2007.

[28] D. Hu, "Detoxic action of acupoint-injection in chemotherapy of malignant tumor," Chinese Journal of Acupuncture and Moxibustion, vol. 23, no. 10, pp. 587-588, 2003.

[29] M. Tao, M. Lai, and H. Lu, "A Clinical observation of the water injection treatment for vomiting during tumor chemotherapy," Chinese Journal of Nursing, vol. 35, no. 8, pp. 462-464, 2000.

[30] WHO, WHO Handbook for Reporting Results of Cancer Treatment, World Health Organization, Geneva, Switzerland, 1979.

[31] S. Chen, J. Chen, Z. Fan, L. Meng, J. Xu, and W. Niu, "Effect of gastrointestinal functional rehabilitation in rectal cancer after Miles operation treated by injection of neostigmine under time medicine mode," Hebei Journal of Traditional Chinese Medicine, vol. 32, no. 6, pp. 883-884, 2010.

[32] W. Feng, C. Xiao, and Z. Huang, "Acupoint injection of vitamin B1 for post operative intestinal function recovery of colorectal cancer patients," Family Nurse, vol. 5, no. 11, pp. 32-33, 2007.

[33] H. Sui and H. Zhang, "Efficacy of aqua acupuncture treatment for intractable hiccup associated with maligment tumor," Chinese Journal of Misdiagnosis, vol. 9, no. 30, pp. 7360-7361, 2009.

[34] H. Xia, H. Ma, and H. Pang, "Acupoint injection associated with the treatment of intractable hiccup of cancer patients," Chinese Folk Medicine, vol. 8, no. 5, p. 12, 2000.

[35] J. Yan, C. Zhou, G. Li, and C. Lu, "Efficacy of recombinant human interleukin-2 injectiona at acupoint for fever from cancer," Chinese Acupuncture and Moxibustion, vol. 7, pp. 415416, 1999. 
[36] J. Yan, D. Yang, R. Zhao, and G. Li, "Zusanli injection treament for persistent fever of upper gastroinstestinal malignant tumor," Chinese Acupuncture and Moxibustion, vol. 11, pp. 687-688, 1997.

[37] Q. Xue, "Clinical observation of astragalus acupoint injection for treatment of advanced malignant tumor," Traditional Chinese Medicine Journal of Liaoning, vol. 32, no. 12, pp. 1269-1270, 2005.

[38] Y. Zhao, H. Wang, M. Chen, and P. Li, "A clinical observation of acupoint injection for chemotherapy-induced gastrointestinal reaction," Journal of Liaoning University of Traditional Chinese Medicine, vol. 10, pp. 115-116, 2008.

[39] J. Deeks, J. Higgins, and D. Altman, "Chapter 8: assessing risk of bias in included studies," in Cochrane Handbook for Systematic Reviews of Interventions Version 5.1.0, J. Higgins and S. Green, Eds., The Cochrane Collaboration, 2011, http://www.cochrane-handbook.org.

[40] J. Deeks, J. Higgins, and D. Altman, "Chapter 9: analysing data and undertaking meta-analyses," in Cochrane Handbook for Systematic Reviews of Interventions Version 5.1.0, J. Higgins and S. Green, Eds., The Cochrane Collaboration, 2011, http://www.cochrane-handbook.org.

[41] J. P. T. Higgins and S. G. Thompson, "Quantifying heterogeneity in a meta-analysis," Statistics in Medicine, vol. 21, no. 11, pp. 15391558, 2002.

[42] B. Yanju, L. Yang, B. Hua et al., "A systematic review and metaanalysis on the use of traditional Chinese medicine compound kushen injection for bone cancer pain," Supportive Care in Cancer, vol. 22, no. 3, pp. 825-836, 2014.

[43] M. K. Garcia, J. McQuade, R. Haddad et al., "Systematic review of acupuncture in cancer care: a synthesis of the evidence," Journal of Clinical Oncology, vol. 31, no. 7, pp. 952-960, 2013.

[44] E. Basch, A. A. Prestrud, P. J. Hesketh et al., "Antiemetics: American Society of Clinical Oncology clinical practice guideline update," Journal of Clinical Oncology, vol. 29, no. 31, pp. 41894198, 2011.

[45] C. I. Ripamonti, E. Bandieri, and F. Roila, "Management of cancer pain: ESMO clinical practice guidelines," Annals of Oncology, vol. 22, supplement 6, pp. vi69-vi77, 2011.

[46] J. P. Ioannidis, S. J. Evans, P. C. Gøtzsche et al., "Better reporting of harms in randomized trials: an extension of the CONSORT statement," Annals of Internal Medicine, vol. 141, no. 10, pp. 781788, 2004.

[47] J. Deeks, J. Higgins, and D. Altman, "Chapter 14: adverse effects," in Cochrane Handbook for Systematic Reviews of Interventions Version 5.1.0, J. Higgins and S. Green, Eds., The Cochrane Collaboration, 2011, http://www.cochrane-handbook.org.

[48] J. Chen, Q. Lv, M. Yu, X. Zhang, and J. Gou, "Randomized clinical trial of Chinese herbal medications to reduce wound complications after mastectomy for breast carcinoma," British Journal of Surgery, vol. 97, no. 12, pp. 1798-1804, 2010.

[49] L. Cheng, "Clinical observation of anisomine with different doses for pain relief," Journal of Mathematical Medicine, vol. 25, no. 6, p. 692, 2012.

[50] J. Kim and D. I. Kang, "A descriptive statistical approach to the Korean pharmacopuncture therapy," Journal of Acupuncture and Meridian Studies, vol. 3, no. 3, pp. 141-149, 2010.

[51] M. Chao and C. Wade, "P02. 44. A pilot crossover trial of acupoint injection for treating primary dysmenorrhea," $B M C$ Complementary and Alternative Medicine, vol. 12, supplement 1, p. 100, 2012.
[52] J. W. Park, J. H. Jeon, J. Yoon et al., "Effects of sweet bee venom pharmacopuncture treatment for chemotherapyinduced peripheral neuropathy: a case series," Integrative Cancer Therapies, vol. 11, no. 2, pp. 166-171, 2012.

[53] C. Spadacio and N. F. de Barros, "Use of complementary and alternative medicine by cancer patients: systematic review," Revista de Saude Publica, vol. 42, no. 1, pp. 158-164, 2008.

[54] X. Luo and X. Wang, "Application of acupoint injection for clinical oncology," Chinese Journal of Information on TCM, vol. 14, no. 6, pp. 94-96, 2007.

[55] K. F. Schulz, D. G. Altman, and D. Moher, "CONSORT 2010 Statement: Updated guidelines for reporting parallel group randomised trials," BMC Medicine, vol. 8, article 18, 2010.

[56] A. B. Miller, B. Hoogstraten, M. Staquet, and A. Winkler, "Reporting results of cancer treatment," Cancer, vol. 47, no. 1, pp. 207-214, 1981.

[57] H. MacPherson, D. G. Altman, R. Hammerschlag et al., "Revised Standards for Reporting Interventions in Clinical Trials of Acupuncture (STRICTA): Extending the CONSORT Statement," PLoS Medicine, vol. 7, no. 6, Article ID e100261, 2010.

[58] Q. Ning, "Contrast observation on injection metoclopramide in different acupuncture point for the treatment of emesis after chemical treatment to mammary cancer," Hunan Guiding Journal of Traditional Chinese Medicine and Pharmacology, vol. 10, no. 2, pp. 39-41, 2004. 


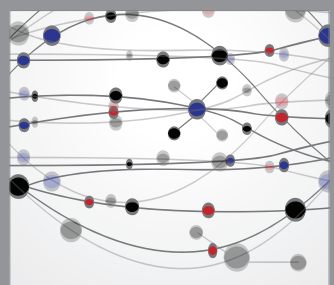

The Scientific World Journal
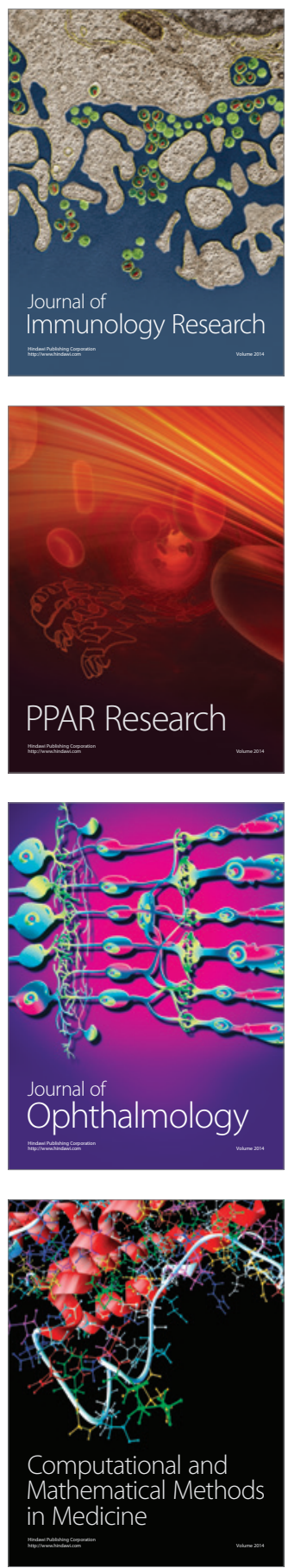

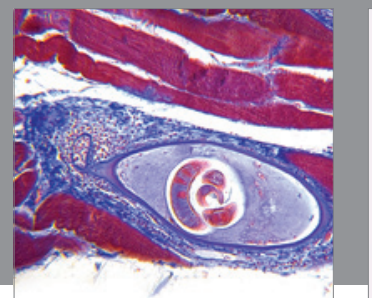

Gastroenterology

Research and Practice
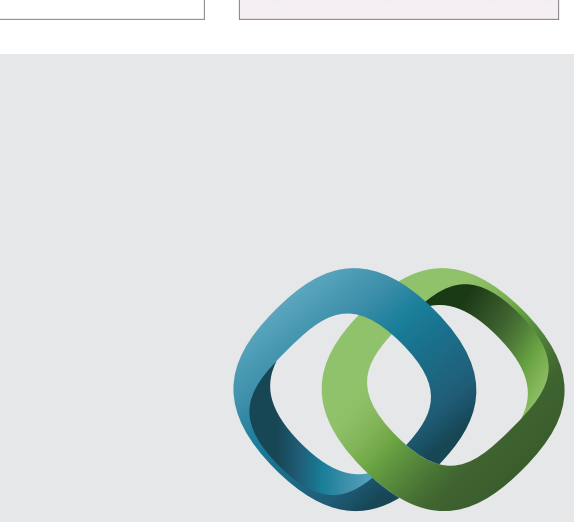

\section{Hindawi}

Submit your manuscripts at

http://www.hindawi.com
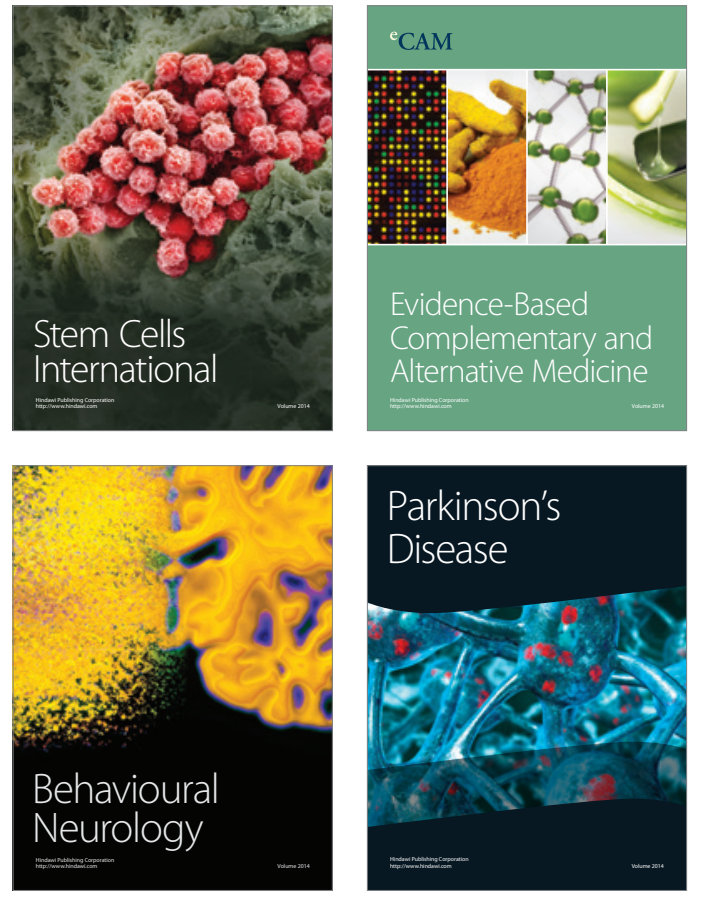
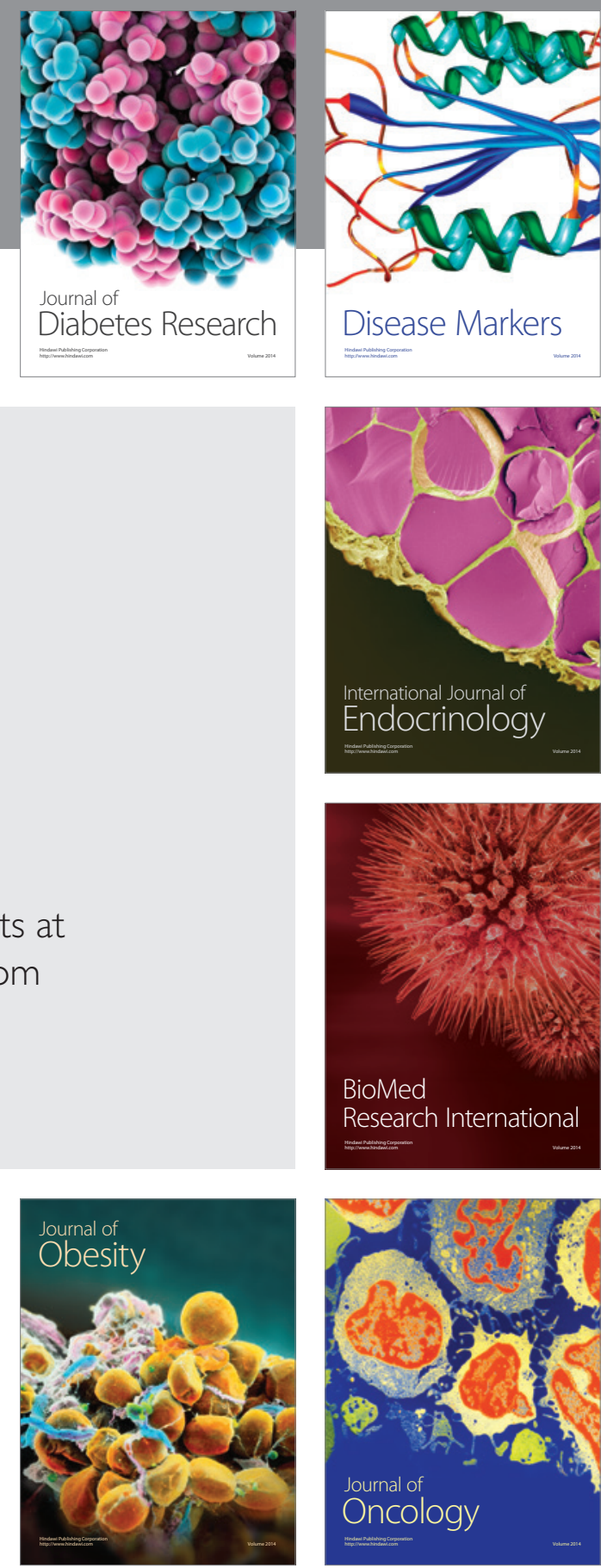

Disease Markers
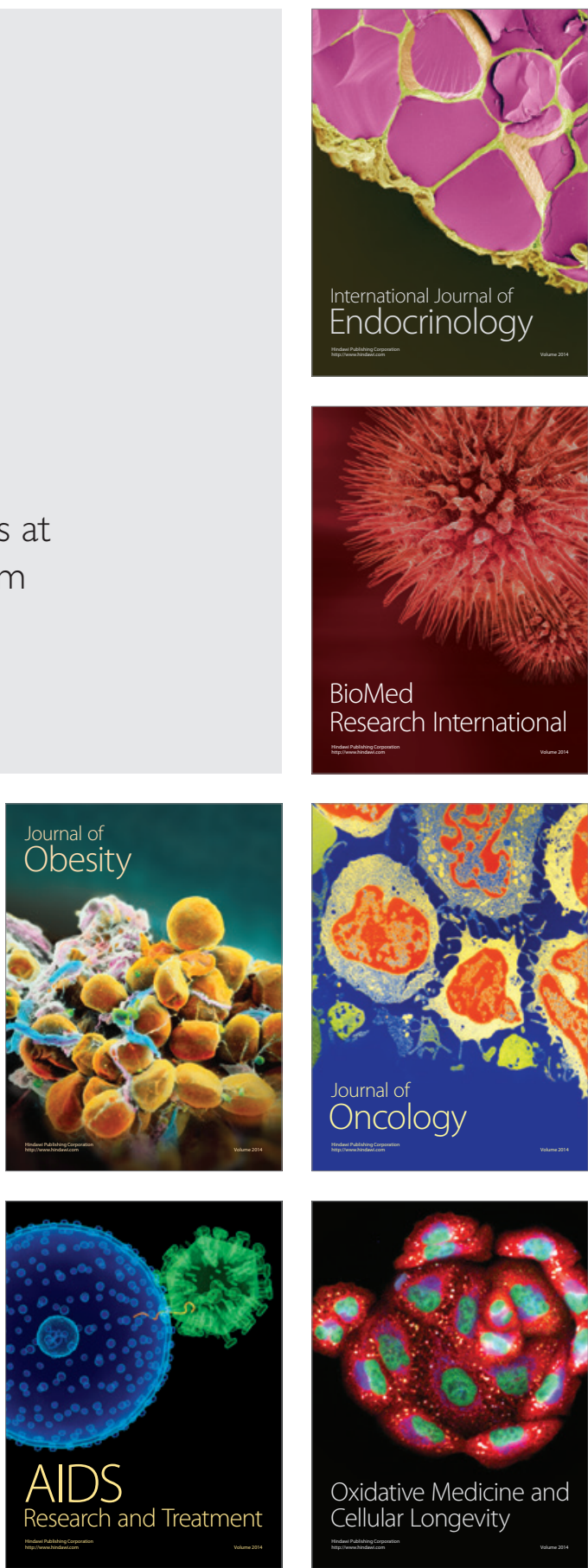